\title{
La introducción de discurso directo por medio del aproximador como en el español de Chile
}

\author{
Lissette Mondaca Becerra ${ }^{1}$ \\ Universitat de València, España
}

\begin{abstract}
Resumen
En la última década, diversos estudios han dado cuenta de la polifuncionalidad de la partícula como en el español. Más allá de su valor semántico aproximador, se ha establecido que como se presenta en el discurso cumpliendo además funciones pragmáticodiscursivas, como retardación, atenuación e introducción de discurso directo (Holmvik 2011; Jørgensen 2012; Panussis 2016; Mondaca 2017; Panussis y San Martín 2017, Mondaca 2019). En el presente artículo se busca analizar, por una parte, la relación que existe entre el uso aproximador de como y su función de introductor de discurso directo en el español de Chile, con el fin de establecer qué tipos de información son introducidos en estilo directo por los hablantes mediante esta partícula. Por otra parte, y de forma exploratoria, se busca identificar las combinatorias sintácticas que presenta como con esta función. Para lograr estos objetivos, se ha utilizado un corpus de 24 entrevistas sociolingüísticas recopiladas en el Proyecto Fondecyt 11110211. La distribución de este corpus contempla seis hablantes por cada una de las cuatro macrozonas geográficas de Chile (Wagner, 1998, 2004). Los principales resultados indican: a) que la función de introducción de discurso directo asociada a como se vincula
\end{abstract}

1 Para correspondencia, dirigirse a: Lissette Mondaca Becerra (lismonbe@alumni.uv.es), Avenida Blasco Ibáñez 32, CP 46010, València, España. ORCID 0000-0001-6057-1911. 
con el valor aproximador de la partícula; b) que el estilo directo introducido por como no solo reproduce citas de habla, sino también pensamientos, discursos hipotéticos, estereotipados y actitudes; y c) que, si bien como puede operar como único elemento introductor de discurso directo, lo más frecuente es que actúe en combinación con otros elementos, como el verbo ser y el adverbio así.

Palabras clave: como, partículas discursivas, aproximadores, discurso directo, español de Chile.

\title{
DIRECT SPEECH INTRODUCTION THROUGH THE APPROXIMATOR COMO IN CHILEAN SPANISH
}

\begin{abstract}
In the last decade, several studies have shown the polyfunctionality of the particle como in Spanish. Beyond its approximating semantic value, it has been established that como also fulfils pragmaticdiscursive functions, such as retardation, mitigation and introduction of direct speech (Holmvik, 2011; Jørgensen, 2012; Panussis, 2016; Mondaca, 2017; Panussis and San Martín, 2017, Mondaca, 2019). This paper aims to analyze, on the one hand, the relationship between the approximating use of como and its function as a direct speech introducer in Chilean Spanish, in order to establish what types of information are introduced in direct style, through this particle, by the speakers. On the other hand, and from an exploratory perspective, the study pursues to identify the syntactic combinations that como, as a direct speech introducer, presents. To achieve these aims, a corpus of 24 sociolinguistic interviews collected in the Fondecyt Project 11110211 has been used. The distribution of this corpus includes six speakers from each of the four geographical macro-areas of Chile (Wagner, 1998, 2004). The main results show the following: a) The function of introduction of direct speech associated to como is linked to the approximating value of the particle; b) the direct speech introduced by como not only reproduces speech quotes, but also thoughts, hypothetical speech, stereotyped speech, and attitudes; and, c) although como can operate as an only introductory element of direct speech, most frequently it acts in combination with other elements, such as the verb ser and the adverb asi.
\end{abstract}

Keywords: como, discourse particles, approximators, direct reported speech, Chilean Spanish.

Recibido: 10/04/20

Aceptado: 04/03/21 


\section{INTRODUCCIÓN ${ }^{2}$}

El discurso directo se define, en términos generales, como aquel que reproduce de forma literal palabras y pensamientos (Nueva gramática de la lengua española [NGLE] 2010). Si bien los verba dicendi han sido reconocidos como los principales elementos introductores de discurso directo y, por ende, han sido las formas más estudiadas en este ámbito, en la oralidad abundan distintas formas introductoras, como la combinación $y+$ frase nominal, verbos no dicendi, la forma nula, entre otras. A estos elementos, hoy podemos añadir además determinadas partículas discursivas que han adquirido este papel (Borreguero 2017; De la Mora 2018). Algunos ejemplos de estas partículas son en plan, en el español peninsular (Jørgensen 2009; Rodríguez 2015; Méndez 2016; Borreguero 2017 y 2020; Grutschus en prensa); onda, en el español de Chile (San Martín, Rojas y Guerrero 2016, Mondaca 2020); así, en el español de México (De la Mora 2018); o tipo, en el español de Argentina (Mihatsch 2018). En otras lenguas, encontramos, por ejemplo, el uso de like, en inglés (Andersen 2001; Levey 2003; Tagliamonte 2009; Jørgensen y Stenstrøm 2009; Holmvik 2011; Jørgensen 2012; Tagliamonte 2016; Panusis y San Martín 2017), o el de tipo, en italiano (Voghera 2013; Borreguero 2020). Este es también el caso del aproximador como.

En la última década, diversos estudios han dado cuenta de las múltiples funciones que cumple la partícula como en el español. Estos trabajos se han propuesto delimitar estas funciones y establecer una categorización de los usos reales que hacen los hablantes de este elemento (Holmvik 2011; Jørgensen 2012; Panussis 2016; Mondaca 2017; Panussis y San Martín 2017; Mondaca 2019, entre otros). En dichos estudios se ha llegado a la conclusión de que como presenta en el discurso funciones que van más allá de su valor de aproximador semántico (hedge), y que su uso se relaciona también con funciones discursivas ${ }^{3}$. Así, todos estos trabajos coinciden en que una de las funciones de la partícula como en el discurso oral es introducir estilo directo.

\footnotetext{
2 La realización de este estudio ha sido posible gracias al financiamiento de la Agencia Nacional de Investigación y Desarrollo (ANID), Gobierno de Chile, a través de su Programa de Formación de Capital Humano Avanzado, Becas de Doctorado en el Extranjero/2018 72190244 .

3 Atenuación, retardación, aproximación numérica, ejemplificación, introducción de discurso directo.
} 
Veamos un ejemplo:

1) M6A2: Entonces, como que tengo otra manera de vivir la vida y pensar la vida y mi mamá con mi hermana son mucho máh cuadra'itas, entonces siempre chocamos y chocamos de manera fea, pero mi tata es como el conciliador de la situación, como "ya, si te sientes mal, conversemos, si te sientes mal, salgamos", él pesca su muleta miércale y salimos ahí, a dos por hora, pero-(risas), pero me habla, me conversa, o de repente el solo hecho que tiene eso de que mete la mano a su bolsillo y te mete pastillas adentro de tus bolsillos.

En 1) podemos observar el uso de como como un recurso que la hablante utiliza a la hora de introducir un discurso directo usualmente enunciado por su abuelo en una situación estereotipada para su entorno familiar. Ahora bien, si seguimos la definición de las gramáticas acerca del estilo directo, la introducción de este tipo de discurso mediante un aproximador hace que nos preguntemos si efectivamente podemos reconocer el discurso reproducido como literal. Ante esto, postulamos que como, al ser un elemento aproximador, permite a los hablantes introducir la reproducción de lo dicho o pensado - por ellos mismos o por otras personas- de una forma que pareciera ser directa, es decir, literal, pero que, sin embargo, es aproximada. De esta forma, como vendría a constituir una marca discursiva que avisa al interlocutor que el discurso citado a continuación no es del todo exacto.

Así, por una parte, en el presente artículo buscamos analizar la relación que existe entre el valor aproximador de como y su función de introductor de discurso directo en el español de Chile, partiendo de la hipótesis de que su uso con esta función deriva de su anterior función aproximadora (Mihatsch 2010; Mondaca 2019). Asimismo, dado que ha llamado nuestra atención que en los diversos trabajos en que se ha identificado el uso de como como introductor de discurso directo no se proponga una clasificación de los tipos de información reproducidos que se pueden introducir mediante esta partícula, sino que solo se mencionen estos tipos a modo general ${ }^{4}$, buscamos también proponer una categorización detallada de los tipos de información introducidos por como en su uso como introducción de discurso directo. Finalmente, un último objetivo se relaciona con estudiar, de forma exploratoria, con qué elementos del discurso como es frecuentemente combinado a la hora de introducir estilo directo, dado que los datos de nuestro corpus sugieren que, al emplearse con la función que hoy estudiamos, como

4 Citas, interjecciones, gestos, pensamientos y sonidos. 
suele presentarse en conjunto con otras partículas que contribuyen también a la introducción de discurso.

En los apartados que siguen, revisaremos brevemente aspectos teóricos sobre el discurso directo y sobre la partícula como como aproximador semántico. Luego, expondremos el corpus de análisis y la metodología utilizados en este trabajo, para, posteriormente, presentar los resultados, las principales conclusiones y algunas posibilidades de ampliación del estudio.

\section{MARCO CONCEPTUAL}

\subsection{DisCURSO DIRECTO}

La Nueva gramática de la lengua española define el discurso directo como aquel "que reproduce de forma literal palabras o pensamientos" (NGLE, 2010: 833). Se ha planteado, desde la normatividad, que el discurso directo se vincula siempre con la reproducción exacta de lo dicho o pensado; no obstante, en diversos estudios se ha argumentado que el discurso directo no es literal, sino que solo se presenta como si lo fuera (Portolés 2004; Herrero Cecilia 2006; Camargo 2007-2008; Gallucci 2012a; San Martín y Guerrero 2013, López Alonso 2014; Gallucci 2016).

En este sentido, Camargo (2007-2008: 56) considera, por ejemplo, que "prácticamente todas las citas que surgen en la interacción conversacional son representaciones o tipificaciones meramente aproximativas e icónicas [...]". Así también, por su parte, López Alonso (2014: 56) postula que "el discurso directo $[\ldots]$ es la reproducción literal de palabras propias o ajenas, con independencia de que esos enunciados sean reales o ficticios, exactos o aproximados". De esta forma, si bien el discurso directo se presenta como literal, esta literalidad no sería exacta, sino aproximada.

En cuanto a algunas formas de introducir estilo directo, se ha señalado que este tipo de discurso "suele aparecer con un verbo introductor que ocupa diversas posiciones" (NGLE 2010: 833). Sin embargo, si bien los verba dicendi son considerados los principales elementos introductores de discurso directo, en la oralidad, principalmente, existen numerosos casos donde se omite el verbo introductor (Gallucci 2012b, 2014, 2016; San Martín y Guerrero 2013; De la Mora 2018) y son otros rasgos -prosódicos, por ejemplo- los que dejan entrever la inserción de un discurso directo. En el siguiente ejemplo es posible observar, primero, el uso de un verbo dicendi 
$\mathrm{y}$, luego, de la forma nula al momento de introducir un discurso reproducido en estilo directo (2):

2) M6A1: Raja'o mi tío. Y la otra vez, la última vez que fui-oh, si mi tío es bacán, eh, me dijo, "toma, esto es para Juan Pablo", "ya, ¿invitación de algo?", le dije, "no, que él lo vea”, y era un sobre.

Además de estas formas, clasificadas por De la Mora (2018) como canónicas, existen otras formas no canónicas que permiten introducir este tipo de discurso, por ejemplo, se ha identificado la construcción $y+$ frase nominal $^{5}$ (3) (Cameron 1998; San Martín y Guerrero 2013; De la Mora 2018) y la partícula así (4) (De la Mora 20186), formas introductoras de estilo directo que hoy nos resultan de gran interés, debido a que, además de también ser utilizadas en el español de Chile, son formas que, a menudo, se combinan con la partícula como a la hora de emplearse con esta función. Nos referiremos a esto con mayor detalle en los resultados de este trabajo.

3) Entrevistador: ¿Y eran todos chicos?

H2A2: Sí poh, todos chicos y el Pelao me dice "colócate el cinturón, colócate el cinturón" y yo "ya, calma'o, si no pasa nada"

4) M4A4: Ya y eso, estaba enojado conmigo poh. No hablamos -de hecho, me acuerdo que ese año, a fin de año lo operaron del codo. Una hueá así

Entrevistador: Ah sí poh, la operación del codo

M4A4: Y yo le escribí. Le postié asi "oye, que te vaya bien" porque yo no-él fue el que se enojó conmigo poh, no yo con él y el hueón llegó y borró el mensaje (risas)

Como hemos señalado en el apartado introductorio, existen partículas discursivas que han sido reconocidas como mecanismos de introducción de discurso reproducido. A este respecto, Borreguero (2017) postula que existen partículas que se han especializado en indicar la transición al discurso directo en la interacción "y que dichas partículas asumen la función de marcas textuales del discurso citado, sustituyendo a veces incluso al verbo dicendi que habitualmente lo introduce" (Borreguero 2017: 63). Como, así, es un

5 San Martín y Guerrero (2013) identifican esta forma para el español de Chile y la denominan Discurso Directo con Pronombre Personal.

6 Estudio centrado en el español de México. 
ejemplo de aquellas partículas discursivas que han adquirido la función de introductores de estilo directo.

Hemos revisado hasta aquí aspectos generales sobre la introducción de discurso directo. En el apartado siguiente, nos enfocaremos en describir el valor aproximador de como y su vinculación con la función discursiva de introductor de discurso directo que presenta en la oralidad.

\subsection{COMO APROXIMADOR E INTRODUCTOR DE DISCURSO DIRECTO}

Estudios de carácter diacrónico del aproximador como han determinado que su valor como difuminador de significado deriva de un anterior uso prepositivo que indica comparación de similitud (Mihatsch 2009, 2010, 2020). La comparación de similitud, a diferencia de la ecuativa que "establece una identidad de grado o de cantidad" (Mihatsch 2010: 179), expresa una similaridad global entre las identidades o procesos comparados ${ }^{7}$. Veamos algunos ejemplos de comparación ecuativa (5), de similitud (6) y de aproximación (7) mediante como:

5) Eran tan simpáticos como inteligentes ${ }^{8}$.

6) Es como su madre9.

7) M4A4: Ah, es que no sé muy bien, pero trabaja como en el área de comunicación, informática, en fin $[\ldots]^{10}$.

En 5) observamos una comparación ecuativa, en tanto que las cualidades comparadas están al mismo nivel y los dos elementos de la comparación se encuentran explícitos. En 6), por su parte, estamos ante una comparación de similitud, puesto que como marca la existencia de una relación de semejanza entre dos referentes (el hijo o la hija y su madre). Finalmente, en 7) estamos ante un uso aproximador de la partícula como, puesto que marca una aproximación a una semejanza global. Así, el área de "comunicación, informática" sería un campo que guarda una similitud aproximada con el área específica a la que busca hacer referencia la hablante; sin embargo, no hay

\footnotetext{
Mihatsch 2010: 179, citando a Haspelmath y Buchholz (1998).

8 Ejemplo extraído del Diccionario de la lengua española. No se han encontrado ejemplos de este tipo de comparación en el corpus de análisis.

9 Ejemplo extraído de Mihatsch (2010: 177).

10 Ejemplo extraído de Mondaca (2019: 33).
} 
otro elemento explícito en el discurso con el que se realice la comparación (Mondaca 2019).

El uso de como como aproximador semántico, uso derivado entonces de la comparación de similitud, ha servido como punto de partida para nuevas funciones, ya pragmático-discursivas, de la partícula (Mihatsch 2010). Estas funciones son la aproximación numérica, la ejemplificación, la retardación, la atenuación y la introducción de discurso directo (Mihatsch 2010; Holmvik 2011; Jørgensen 2012; Panussis 2016; Mondaca 2017; Panussis y San Martín 2017; Mondaca 2019). En lo que respecta a la función de introducción de discurso directo de como, esta ha sido identificada para el español de Chile en los trabajos de Jørgensen (2012), Panussis (2016), Mondaca (2017), Panussis y San Martín (2017) y Mondaca (2019).

Finalmente, en relación con qué tipos de información son introducidos en estilo directo por medio de como, los estudios que hemos revisado establecen que permite introducir citas, pensamientos, interjecciones, gestos y sonidos (Holmvik 2011; Panussis 2016; Mondaca 2017; Panussis y San Martín 2017; Mondaca 2019). Sin embargo, nuestro corpus nos permite postular que como, además, permite introducir cuestiones que van más allá de la mera reproducción de palabras y que se relacionan directamente con aspectos pragmáticos, por ejemplo, actitudes, sensaciones y sentimientos de los hablantes en relación con lo que reproducen. Ahondaremos en este planteamiento en los resultados del trabajo.

Hemos realizado hasta aquí un breve recorrido por la bibliografía que ha identificado el valor aproximador de como y la vinculación de esta partícula con la introducción de discurso directo. A continuación, describimos el corpus con el que hemos trabajado y la metodología aplicada.

\section{CORPUS DE ANÁLISIS Y METODOLOGÍA}

Con el fin de analizar el uso de como como partícula introductora de discurso directo, hemos trabajado con un corpus de 24 entrevistas sociolingüísticas ${ }^{11}$, recopiladas en el Proyecto El voseo en Chile: Un cambio lingüístico en

11 Cada entrevista tiene una duración aproximada de una hora. 
desarrollo. Aspectos internos y externos de la variación ${ }^{12}$. Las entrevistas, recopiladas entre los años 2011 y 2014, cuentan con la particularidad de abarcar el habla de todo el territorio chileno, que se distribuye en cuatro macrozonas geográficas (Wagner 1998, 2004) -seis entrevistas por cada zona-. Así, el análisis se ha realizado sobre una muestra de habla que nos permitirá estudiar el uso de como como introductor de discurso directo a nivel nacional. En su totalidad, el corpus suma 172993 palabras.

De nuestro corpus, hemos extraído y analizado manualmente todos aquellos contextos donde hemos identificado el uso de la partícula como con la función que hoy estudiamos, que suman 130 casos. La metodología para la identificación de los contextos ha sido definida sobre la base del propio análisis de los datos. Los principales criterios que han servido de guía para reconocer la función analizada han sido la exclusión de los usos comparativos, el uso de comillas, el empleo de como junto al verbo decir ( $u$ otros verbos que han adquirido el valor de citación, como quedar); el uso de la partícula en construcciones como $y+$ frase nominal/pronombre como; las locuciones ser como y así como, identificadas previamente como combinatorias frecuentes para el uso de como como introductor de discurso directo (Panussis y San Martín 2017), entre otros elementos contextuales.

Sobre la base de la exploración realizada al corpus considerando los criterios mencionados anteriormente, y tomando como referencia lo mencionado en trabajos previos sobre contenidos introducidos por como en estilo directo (Holmvik 2011; Jørgensen 2012; Panussis 2016; Mondaca 2017; Panussis y San Martín 2017; Mondaca 2019), los principales tipos de información introducidos por como corresponderían a:

a) Habla directa: reproducción aproximada de palabras propias o ajenas emitidas en un contexto pasado real.

b) Pensamientos: reproducción aproximada de pensamientos pasado o presentes -discursos no emitidos en la realidad- de los propios hablantes.

c) Discurso estereotipado: reproducción aproximada de un discurso generalizado o ejemplificado propio o ajeno, pasado o presente, que se da con mayor o menor frecuencia en situaciones estereotipadas.

d) Discurso hipotético: reproducción aproximada de un discurso no emitido en una situación real, propio o ajeno, imaginado para una situación pasada o futura.

12 Proyecto financiado por el Fondo Nacional de Desarrollo Científico y Tecnológico (Fondecyt - 11110211). 
e) Interjecciones: reproducción de exclamaciones vinculadas con la aproximación a actitudes, sensaciones y sentimientos en relación con la situación que se describe.

f) Sonidos: simulación aproximada de algún sonido.

Cada uno de estos tipos de información es explicado y ejemplificado en el siguiente apartado, dedicado a los resultados de este trabajo.

\section{RESULTADOS}

El análisis realizado nos permite establecer y reafirmar el uso de la partícula como como un elemento introductor de discurso directo en el habla chilena. Con este estudio, hemos podido ratificar el planteamiento existente acerca de que el uso de como como introductor de discurso directo deriva, efectivamente, de su valor semántico aproximador (Mihatsch 2010), ya que esta partícula marca la difuminación del significado del discurso citado que le sigue $^{13}$. De esta forma, la literalidad del discurso que se introduce mediante como no es exacta, sino siempre aproximada.

Hemos comprobado que su uso con esta función permite introducir diversos tipos de información, descritos a continuación, y que tales informaciones pueden referir a discursos pasados, presentes o futuros, propios del hablante o ajenos a él, y reales o ficticios (4.1.). Además, a través del análisis hemos podido dar cuenta de que como puede actuar como única partícula introductora de discurso o en combinación con otros elementos (4.2.).

\subsection{TIPOS DE INFORMACIÓN}

Como ya anunciábamos en el apartado metodológico, a través del análisis hemos podido establecer seis categorías principales de tipos de información introducidos en estilo directo mediante como: habla directa, pensamientos,

13 Existen trabajos que, además, plantean un posible origen del uso citativo de como en su valor ejemplificador (véase, Grutschus, en prensa); sin embargo, para estudiar estas posibilidades sería necesario profundizar en la diacronía de la partícula. 
discurso prototípico, discurso hipotético, interjecciones y sonidos. Presentaremos en detalle cada una de estas categorías y proporcionaremos ejemplos para cada caso:

a) Habla directa: Reproducción aproximada de palabras propias o ajenas emitidas en un contexto real, específico y puntual, del pasado.

8) M6A1: Y de ahí el caballero que me decía antes, me dijo, "no, si cruzaron, cruzaron". Cruzamos y los perdimos. "Se metieron al mercado", decía "se metieron al mercado". Y ahí nos quedamos en la esquina y a mí me dio rabia, porque a la señora que la habían asaltado y le habían quitado, no hacían nada, estaba como, "uy, ¿qué voy a hacer? Me robaron. Mi monedero tenía plata, oh, ¿qué voy a hacer?", y nada máh. Y yo, “oh, esta señora me tiene aburrí'a, así es que le dije, "caballero, ¿sabe qué máh?, acompáñeme", le dije, "vamos a buscar a estos delincuentes", "ya poh", me dijo y el caballero me pesca el brazo, miércale, y me lleva.

9) M2A6: Y me asusté. Decía, "Se están entrando por la ventana", y yo, “Ay, ¿qué pasa?”, ya, así como, “ya, voy a ir a ver”. Me acerqué y no había nada poh, estaba alucinando, pero en esas oportunidades yo me asusté, como que me llamaba a cada rato-me llama a cada rato cuando le dan esas cosas, porque yo creo que lo que ella me transmite es que le da miedo, le dan miedo todas las cosas que ve.

En 8), la informante relata la experiencia que vivió cuando casi fue víctima de un robo en las calles de una ciudad chilena; le cuenta a la entrevistadora que, a diferencia de una mujer a la que sí le habían robado y solo se queda lamentando lo sucedido, ella se atrevió a seguir a los ladrones. Mediante como, la hablante introduce una aproximación al habla directa de la mujer asaltada. En 9), por su parte, la hablante relata la experiencia que vivió con su abuela, quien padece Alzheimer, cuando esta imaginó que había gente que quería entrar a robarle por la ventana de su habitación. Así, mediante como, la informante introduce su propio discurso directo al reproducir de forma aproximada lo que le dijo a su abuela en ese momento.

Ambos ejemplos dan cuenta del uso de como como introductor de habla directa, puesto que el discurso reproducido remite al habla real en situaciones pasadas. Sin embargo, también en estos ejemplos podemos observar el valor aproximador de como, en tanto que, con su empleo, ambas hablantes reproducen habla directa de una manera que pudiera considerarse literal, pero que en realidad, es aproximada, pues como actúa como introductor y difuminador de del discurso reproducido. 
b) Pensamientos: Reproducción aproximada de pensamientos de los propios hablantes. En esta categoría de contenido, encontramos cuatro tipos diferentes de pensamiento:

i. Pensamientos pasados que no fueron emitidos por los hablantes en las situaciones a las que refieren. Este es el caso del ejemplo 10), donde la informante relata cómo escogió el nombre que le pondrá a su hija si algún día llega a ser madre de una niña. Al recordar esta situación, reproduce el pensamiento que tuvo en el momento en que decidió que ese era el nombre indicado e introduce la reproducción aproximada de ese pensamiento por medio de como:

10) M4A6: No, no realmente, de hecho, ese nombre lo saqué porque había una dibujante de comics que me gustaba y se llamaba Danae. Entrevistador: Tiene como nombre de comic (risas).

M4A6: Claro, Danae [...] se llamaba la artista ahí me encantó ese nombre, fue como "ah, ya, bacán"14", así. Le voy a poner a mi hija así $\mathrm{y}$ me he acordado todos los años, lo tengo anota'o en la mano (risas).

ii. Pensamientos que tienen los hablantes en su presente ante situaciones que se pueden dar con mayor o menor frecuencia, como lo es el caso de 11), donde el hablante le cuenta a la entrevistadora que, cuando estudiaba, vivía lejos del colegio y que aprovechaba los viajes en autobús, desde y hacia su casa, para estudiar y hacer sus deberes. Ahora, que ya es profesional, guarda aún la idea de que debe estudiar en el autobús, por lo que introduce este pensamiento que se da en su presente por medio como:

11) H4A3: Como los niños. No, todo mi estudio fue en base a la micro ${ }^{15}$ y yo creo que si no es porque me demoro una hora no estaría acá Entrevistadora: No sería quien soy

H4A3: No, en serio, al colegio era como mi hora de estudio en la micro, así como que me sentaba pa' estudiar. [...] Si no estudiaba en la casa, me sentaba y sacaba mi cuaderno y me ponía a subrayar, a hacer las tareas. Ahora no tanto, ahora duermo no más. Es como "tengo que estudiar, no, ya no".

\footnotetext{
14 Bacán: (adj.) muy bueno o muy bien (Diccionario de uso del español de Chile 2010: 104).

15 Micro: (f.) autobús de transporte colectivo urbano (Diccionario de uso del español de Chile 2010: 589).
} 
iii. Pensamientos propios del hablante pero que, a su vez, son compartidos con alguien más. En 12) la informante habla sobre la responsabilidad que tienen ella y su hermana en el cuidado de su abuela materna. Le cuenta a la entrevistadora sobre su rutina diaria y de cómo ellas se turnan para cuidarla durante el día. En el ejemplo, podemos ver cómo la hablante introduce, mediante como, una aproximación al pensamiento que comparte con su hermana cada vez que terminan la rutina de cuidados con su abuela.

12) M2A5: O retando a alguien, cuando no le gusta que alguien-de repente se imagina parientes y los tiene que echar mi hermana poh, y los tiene que echar así y decirles, "No, váyanse de acá, váyanse de mi casa" (risas). Y yo me muero de la risa y le digo, "Javiera, [...] no está, dile que no está. ¿Pa' qué vai a hacer todo el show?", "Porque o si no, me va a decir que sí está y me va a seguir molestando. Y al rato de nuevo, te hace echar varias veces lo mismo, entonces, nos vamos turnando, hasta que después le damos las pastillas para dormir y ahí duerme toda la noche y nosotras como "ya, descansamos hasta mañana". Después en la mañana empieza de nuevo a las seis de la mañana, todos los días.

iv. Pensamientos pasados o presentes que, más que reproducir un discurso no emitido en la realidad, dan cuenta de determinadas actitudes de los hablantes frente a situaciones particulares. Este es el caso de 13), donde la conversación se desarrolla en torno a la experiencia que tuvo la informante de estudiar por un tiempo en una ciudad más grande que su ciudad natal y relata que le gustaría volver allá porque su ciudad le parece aburrida. En este ejemplo, mediante como, la hablante introduce un pensamiento que, más allá de reproducir solo reproducir su pensar, deja entrever una aproximación a la actitud de disgusto y resignación que demuestra en relación con las pocas actividades recreativas que se pueden realizar en la ciudad donde reside.

13) M2A5: Hay máh lugares pa' salir, pa' visitar, no te aburre Entrevistador: Ya

M2A5: Acá sí, es como "ya, Zofri ${ }^{16},{ }^{*}$ mall ${ }^{17 "}$. El *mall en quince minutos recorrido, la universidad queda acá, está al lado, está todo al lado.

Acortamiento para Zona Franca de Iquique.

El asterisco marca el uso de un extranjerismo por parte de la hablante. 
Los ejemplos anteriores ilustran el uso de como como un elemento que permite introducir pensamientos. Consideramos que el hecho de que estos pensamientos sean introducidos por medio de como se debe a que los hablantes buscan marcar que lo que señalan está siendo expresado de forma aproximada, como una forma de verbalizar lo más acertadamente posible lo que pensaron o piensan en una determinada situación.

c) Discurso estereotipado: Reproducción aproximada de un discurso generalizado o ejemplificado, propio o ajeno, pasado o presente, que ocurría o que ocurre con mayor o menor frecuencia en situaciones estereotipadas. Estos discursos pueden identificarse en contextos que se relacionan, por ejemplo, con la familia, el trabajo, los estudios, los amigos, entre otros, $\mathrm{e}$, incluso, en contextos donde los hablantes reproducen un discurso que se puede generalizar para la sociedad chilena. En esta categoría, podemos encontrar tres subtipos:

i. Discurso estereotipado del propio hablante ante una situación pasada o presente que se repetía o repite con mayor o menor frecuencia. Este es el caso de 14), donde la informante le cuenta a la entrevistadora cómo es convivir con su abuelo, con quien tiene una relación muy cercana. Relata que, en ocasiones, su abuelo se levanta a desayunar a las cuatro de la mañana y que la despierta para que desayune con él. Ante esto, la hablante reproduce su propio discurso, un discurso que se hace estereotipado pues se repite en las ocasiones que se da esta situación.

14) M6A2: Tiene ochenta y siete años, pero tiene cada historia y hace cada tontera. De repente ${ }^{18}$ se levanta a las cuatro de la mañana y me dice, "Belén, tengo hambre", y es como, "tata, son las cuatro de la mañana", "ah, no sé, yo me acosté a las siete, yo pensaba que había que tomar desayuno", y él toma desayuno a las cuatro de la mañana y me hace comer a las cuatro de la mañana (risas).

ii. Discurso estereotipado ajeno que se repite con frecuencia en determinados contextos. En el ejemplo 15), la conversación versa sobre que la hablante es aficionada a jugar videojuegos en línea y que, cuando lo hace, prefiere no decir que es mujer, pues generalmente quienes juegan en línea son hombres y estos creen que las mujeres no son buenas jugadoras. Así, por medio de

18 Indicador de ocurrencia esporádica. Rojas (2008: 217) clasifica este uso de de repente como el de "locución adverbial de frecuencia, equivalente a los sintagmas 'a veces', 'de vez en cuando', 'en ciertas ocasiones"'. 
como, la informante introduce una aproximación al discurso prototípico de los jugadores hombres.

15) M4A6: Yo jugaba The Soldiers y bajaba mapas para subir, escalar, todo [...] proyectiles. Como esos típicos proyectiles que uno salta y tiene que disparar bien. Habían mapas donde los monos saltaban y todo, pero parece que tú teníai que matarlos de distintas maneras.

Entrevistador: [...] así un juego de meterme y hueviar, todo mal así.

M4A6: Claro, no, es que a mí igual me pasaba que de repente cuando se daban cuenta de que era mujer, tampoco me gustaría que dijeran como "ah, es mujer, mejor no juguemos con ella porque debe ser mala".

iii. Discurso estereotipado compartido por o atribuido a la sociedad chilena en general, como en el caso de 16), donde el hablante relata que conoció a alguien que proviene de La Legua, un barrio estigmatizado socialmente de la comuna de San Joaquín, Región Metropolitana de Chile. La estigmatización de este barrio es conocida por la sociedad chilena en general. Ante esto, el hablante señala que, al conocer a esta persona, se quitó el estigma que tenía de quienes viven allí y, por medio de como, introduce una pregunta prototípica que se le haría a alguien que proveniente de dicho barrio:

16) H6A6: Anda de todo en realidad, de todo así. La última vez conocí a un loco de La Legua así y la hueá era terrible así como-hablaba así como sha igual. El loco era súper buena tela, hueón.

Entrevistador: Qué bacán.

H6A6: Como que igual me saqué todo el estigma de los locos de allá así.

Entrevistador: Sí poh, como el prejuicio que uno tiene a vecesH6A6: Sí poh, o sea, que en realidad, en general, todos tienen poh. Así como "Ah, ¿veníh de allá?". O sea, el loco igual no tenía el aspecto así de un gallo malacatoso, pero igual poh, onda, su forma de hablar así era diferente poh.

Entrevistador: Claro.

De esta forma, por medio del como los hablantes chilenos introducen en sus discursos otros discursos que pueden considerarse estereotipados, ya sea en el pasado o en el presente, dada la frecuencia con la que se presentan en las situaciones a las que remiten. Nuevamente consideramos que el empleo de como como marca introductora del estilo directo para estos contenidos permite evidenciar la aproximación al discurso reproducido. 
d) Discurso hipotético: Reproducción aproximada de un discurso no emitido en una situación real, propio o ajeno, imaginado para una situación pasada o futura. Este discurso se genera en la situación de enunciación actual, es decir, es un discurso que el hablante hipotetiza en una situación presente y que lleva a situaciones pasadas o futuras. En esta categoría de discurso directo introducido por como, podemos encontrar dos tipos:

i. Discurso hipotético ajeno donde los hablantes imaginan lo que alguien más pudo haber dicho o podría decir en una situación determinada. En el ejemplo 17), la conversación se desarrolla en torno a que la entrevistadora está estudiando pedagogía y que la hermana del informante ya es profesora. La entrevistadora le comenta sobre algunas malas experiencias que ha tenido en sus prácticas en un colegio. El informante, sin ser pedagogo, dice no comprender cómo su hermana tiene paciencia para trabajar con niños. Así, por medio de como, el hablante introduce un titular hipotético de un periódico - discurso ajeno-que haría alusión a un comportamiento que podría tener él si trabajara como profesor, dada su falta de paciencia.

17) Entrevistadora: $Y$ ahí era un colegio chico, como San Bernardo (risas), [...] y está llenos de cerros así, entonces, era un colegio chico y los alumnos no tenían disciplina. No, definitivamente no. Así que lo pasé mal, sufrí

H4A3: Yo digo, mi hermana cómo soporta así. Saldría en el diario al tiro como "profesor... (risas)

Entrevistadora: Golpea al alumno

H4A3: ... golpea al alumno"

Cabe señalar que en la categoría de discurso hipotético ajeno también hemos incluido la introducción de pensamientos ajenos, puesto que, a diferencia de los pensamientos propios, donde los hablantes se citan a sí mismos, los pensamientos que los informantes asignan a terceras personas son imaginados, supuestos, dado que no pueden tener certeza de lo pensado por alguien más:

18) H2A3: Eh, y es como súper cuática con la alimentación. Si sirve unos-pucha, la mayoría de los niños, cuando van a mi casa a almorzar así y ven, mi mamá sirve así unos platos de comida poh. Así y los otros niños ${ }^{19}$ quedan como "¿qué onda? ¿Por qué tanta comida?”. 
Yo estoy acostumbrado, porque yo como no máh poh, y después los otros niños, "no, es que era mucho", y dejan.

En 18), el informante, mediante el empleo de como, introduce el supuesto pensamiento que tienen sus amigos cuando van a almorzar a su casa y su madre les sirve mucha comida. Este pensamiento nace de la actitud de sorpresa que el informante ve cuando sus amigos almuerzan en su casa y que verbaliza a través del verbo quedar, que remite a un estado. Así, este ejemplo constituye un caso de discurso hipotético relacionado con el pensamiento de otros. Quisiéramos mencionar también que este caso podría también haberse clasificado como un ejemplo de discurso estereotipado, pues, contextualmente, podemos saber que los amigos del informante van a comer a su casa con frecuencia. No obstante, dado que el discurso que introduce el informante parece remitir a un pensamiento y no un discurso que sus amigos hayan emitido en la realidad, consideramos que este ejemplo constituiría un caso hipotético.

ii. Discurso hipotético propio donde los hablantes hipotetizan lo que ellos mismos pudieron haber dicho o podrían decir en una situación determinada. En 19) la informante relata la mala experiencia que vivió con sus compañeros de carrera al tener una asignatura donde su madre era también su profesora. La hablante le cuenta a la entrevistadora que, cuando sus compañeros tenían bajas calificaciones en los exámenes, le pedían que ella hablara con su madre para que les diera una oportunidad de subir sus calificaciones; algo con lo que ella no estaba de acuerdo. Así, por medio de como, introduce una pregunta ficticia que podría haberle hecho a su madre, pero que en realidad no lo hizo pues no le parecía lo adecuado.

19) M2A5: Sí y yo así, "yo no tengo nada que ver. Es el trabajo de ella, ustedes tienen que hablar con ella. Yo qué me voy a meter". No, y a veces se hablaban cosas, se metían en cosas de mi mamá, entonces, yo decía, “¿Sabíh qué? Si tú eres capaz de hablar cosas por fuera, anda a hablar con mi mamá y pídele tú ayuda para la prueba" Entrevistador: Ya. Sí poh

M2A5: "Si es tu responsabilidad. Si tú te lo estái echando, ¿cómo voy a estar yo como mensajera? Así como 'mamá, ¿podíh hacerle otro examen a tanto?"”

Así, los ejemplos revisados dan cuenta del uso de como como un elemento introductor de discursos hipotetizados, es decir, que buscan reproducir aproximaciones a posibles discursos (ficticios, supuestos o imaginados) que podrían haberse emitido en situaciones pasadas o que podrían emitirse en situaciones futuras. 
e) Interjecciones: Reproducción de exclamaciones vinculadas con la aproximación a actitudes, sensaciones y sentimientos en relación con la situación que se describe. En 20) la informante relata la experiencia que vivió al asistir a un concierto y describe de manera aproximada la sensación que le produjeron los efectos especiales del evento. Así, mediante el aproximador como introduce la interjección “iguah!”, que representa una sensación abrumadora pero impresionante a la vez.

20) M6A3: Sí y habían fuegos artificiales y salía un avión de arriba que chocó con la muralla y salieron fuegos artificiales y fue como ¡guah! En verdad a mí casi me dio, no sé, un ataque. Yo lloraba, lloraba y un niño delante mío se desmayó.

Este ejemplo permite observar, así, el uso de como como introductor de interjecciones. Este uso, según los datos analizados, se relaciona con que los hablantes buscan introducir un elemento lingüístico que les permita transmitir, de forma aproximada, actitudes, sensaciones y sentimientos experimentados en determinadas situaciones.

f) Sonidos: Simulación aproximada de algún sonido. Este es el caso de 21), donde la informante utiliza el aproximador como para introducir la reproducción de un sonido similar al que produce una persona al roncar. Este uso solo ha sido documentado una vez en nuestro corpus; sin embargo, ya nos permite postular que, en el español de Chile, como es un elemento que permite introducir, en forma de discurso directo, sonidos de manera aproximada.

21) M6A2: No, ya me tiene un poco preocupá', porque de repente, cuando ya necesito dormir y descansar en la noche, me voy a la casa de mi pololo y es como, por favor, necesito dormir.

Entrevistador: Claro.

M6A2: Y ronco toda la noche, así como grgrgr. Pero es un relajo tan grande y ahí ya puedo trasnochar cuatro, cinco noches máh, porque o si no, no puedo, mi cuerpo no da.

Todos los ejemplos revisados anteriormente ilustran los diferentes tipos de información que puede introducir como en su función de introductor de discurso directo en el español de Chile. A través de estos usos podemos establecer que concordamos con lo establecido en trabajos anteriores que indicaban que como introduce discursos directos no literales (Holmvik 2011; Jørgensen 2012; Panussis 2016; Mondaca 2017; Panussis y San Martín 2017; Mondaca 2019), además de confirmar nuestra hipótesis acerca de que como opera como una marca difuminadora de la literalidad del discurso 
reproducido y que, por ende, su función de introductor de discurso directo conecta con su anterior valor de aproximador semántico (hedge).

Nuestro estudio es preeminentemente cualitativo; sin embargo, hemos considerado relevante finalizar este apartado comentando algunos datos de carácter cuantitativo. Presentamos, a continuación, una tabla que resume la frecuencia de uso de como en nuestro corpus según el tipo de información que introduce en estilo directo:

\begin{tabular}{|l|c|c|}
\cline { 2 - 3 } \multicolumn{1}{c|}{} & Frecuencia & Porcentaje \\
\hline Habla directa & 29 & $22 \%$ \\
\hline Pensamientos & 32 & $24 \%$ \\
\hline Discurso estereotipado & 36 & $28 \%$ \\
\hline Discurso hipotético & 14 & $11 \%$ \\
\hline Interjecciones & 18 & $14 \%$ \\
\hline Sonidos & 1 & $1 \%$ \\
\hline \multirow{2}{*}{} & $\mathbf{1 3 0}$ & $\mathbf{1 0 0 \%}$ \\
\cline { 2 - 3 }
\end{tabular}

Tabla 1. Frecuencia de uso como según el tipo de información citada que introduce

Los resultados de este análisis nos indican así que como es empleado por lo hablantes chilenos para introducir, principalmente, discursos estereotipados $(28 \%)$, pensamientos $(24 \%)$ y habla directa $(22 \%)$; y, en menor medida, interjecciones (14\%) -vinculadas con actitudes, sensaciones y sentimientos-, discursos hipotéticos (11\%) y sonidos (1\%). Particularmente, de estos resultados nos llama la atención, por una parte, que el porcentaje de uso más alto de como como introductor de estilo directo se vincule con el discurso estereotipado y, por otra, haber identificado el uso de como como introductor de discursos hipotéticos, dado que ambos tipos de información -discurso estereotipado y discurso hipotético-no habían sido reconocidos en trabajos anteriores (Holmvik 2011; Jørgensen 2012; Panussis 2016; Mondaca 2017; Panussis y San Martín 2017; Mondaca 2019).

\subsection{INTRODUCTOR DE DISCURSO DIRECTO: COMO Y SUS COMBINATORIAS}

Nos hemos propuesto como último objetivo estudiar, de forma exploratoria, aquellos elementos con los que como es frecuentemente combinado a la hora de introducir discurso directo. Si retomamos el ejemplo 1), podemos observar el uso de como como única partícula introductora del discurso citado: 
21) M6A2: Entonces, como que tengo otra manera de vivir la vida y pensar la vida y mi mamá con mi hermana son mucho máh cuadra'itas, entonces siempre chocamos y chocamos de manera fea, pero mi tata es como el conciliador de la situación, como " $y$ a, si te sientes mal, conversemos, si te sientes mal, salgamos", él pesca su muleta miércale y salimos ahí, a dos por hora, pero-(risas), pero me habla, me conversa, o de repente el solo hecho que tiene eso de que mete la mano a su bolsillo y te mete pastillas adentro de tus bolsillos.

Ahora bien, aunque hemos comprobado que como puede actuar como única partícula introductora, el corpus analizado nos permite establecer también que lo más frecuente es que como se posponga sintácticamente a otros elementos, evidenciando así la aproximación al discurso reproducido. Así, como suele presentarse o bien en combinación con otras partículas que contribuyen también a introducir discurso o bien con otros elementos con los que, en conjunto, conforman el segmento introductor.

A través de la exploración realizada, por una parte, podemos determinar que como muy frecuentemente se pospone al verbo copulativo ser (es, era, fue $)^{20}$, en su acepción de elemento que permite "afirmar del sujeto lo que significa el atributo" (DLE), conformando, así, una locución que permite introducir discurso directo aproximado $((y) \mathrm{ser}+\mathrm{como})$.

22) H4A1: ...y es como "chiquillos tengo algo que avisarles, su colegio ya no existe"...

23) M2A5: ...así como que yo iba a clases y era como, "ya, ¿qué edad tiene él?"...

24) M4A6: ...y fue como "ahh, hola soy [...]" y ahí empezamos a hablar...

Cabe señalar que, en nuestro corpus, solo hemos encontrado un caso aislado en el que el verbo ser es utilizado como único elemento introductor de discurso citado, por lo que postulamos que este verbo solo puede contribuir a la inserción de un discurso directo si va seguido de una partícula introductora. Consideramos que la combinatoria ser + como deja entrever claramente la relación que existe entre la aproximación por medio de como y su función anterior como comparativo de similitud.

20 El mismo fenómeno ha sido identificado para la partícula like, en inglés, con su combinatoria be like (Jørgensen y Stenstrøm 2009; Tagliamonte 2009 y 2016; Panussis y San Martín 2017). 
Por otra parte, como también suele combinarse con el adverbio así, partícula equivalente a "de esta o de esa manera, de la forma que se acaba de mencionar o que se va a mencionar a continuación" (DLE). Se ha determinado, y nuestros datos también así lo indican, que así es un elemento que permite introducir discurso directo en sí misma (De la Mora 2018); por tanto, postulamos que como, al combinarse con esta partícula, opera como un

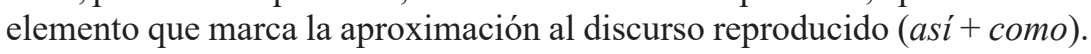

25) H4A3: ¡No te muevas! Mi hermana es así. De repente ella va llegando a su pieza, como que llega a la mía, me cierra la puerta, así como "baja la tele, no hueíh"

26) M6A3: ... hace las pruebas súper difíciles y de memoria, así como "dígame tal y tal cosa"

De esta forma, concordamos con los resultados obtenidos por Panussis (2016) y Panussis y San Martín (2017) para el habla de Santiago de Chile, quienes destacaron la alta frecuencia con la que como se combina con el verbo ser y el adverbio así al emplearse con la función que hoy estudiamos. Además, con nuestro análisis, podemos establecer que estas combinatorias se extienden para el habla chilena en general.

A continuación, presentamos una tabla que incluye todas las combinatorias con las que como se ha presentado en su función de introductor de discurso directo en el corpus analizado:

\begin{tabular}{|l|c|c|}
\hline Combinatoria & Frecuencia & $\%$ \\
\hline ser como & 45 & $34,6 \%$ \\
\hline así como & 36 & $27,7 \%$ \\
\hline como & 13 & $10,0 \%$ \\
\hline (y) pronombre así como & 8 & $6,2 \%$ \\
\hline decir como & 5 & $3,8 \%$ \\
\hline y frase nominal así como & 4 & $3,1 \%$ \\
\hline y pronombre como & 4 & $3,1 \%$ \\
\hline ser así como & 3 & $2,3 \%$ \\
\hline ahí como & 2 & $1,5 \%$ \\
\hline quedar como & 2 & $1,5 \%$ \\
\hline
\end{tabular}




\begin{tabular}{|l|c|c|}
\hline andar así como & 1 & $0,8 \%$ \\
\hline decir algo así como & 1 & $0,8 \%$ \\
\hline decir así como & 1 & $0,8 \%$ \\
\hline estar como & 1 & $0,8 \%$ \\
\hline llegar asi como & 1 & $0,8 \%$ \\
\hline onda como & 1 & $0,8 \%$ \\
\hline quedar así como & 1 & $0,8 \%$ \\
\hline sentir como & 1 & $0,8 \%$ \\
\hline Total & $\mathbf{1 3 0}$ & $\mathbf{1 0 0} \%$ \\
\hline
\end{tabular}

Tabla 2. Combinatorias para como como introductor de discurso directo

La tabla anterior refleja la ya mencionada alta frecuencia de uso de como en combinación con los elementos ser y así. Además, podemos observar que la locución así como se suele combinar también con otros elementos que contribuyen a la introducción de discurso reproducido $((y)$ pronombre asi como (27), y frase nominal así como (27), andar así como, decir algo así como, decir así como, llegar así como, quedar así como. Hemos identificado también casos en los que la locución así como va precedida del verbo ser (ser así como) (28).

27) M2A5: ... y mi mamá así como, "¿por qué no me dijiste? Me cuentan los ayudantes", o no sé qué, y yo así como, "oh, qué lata".

28) M6A2: ... porque en primero yo igual me acuerdo que era así como, "oye, hay que estudiar, hay que estudiar".

La tabla muestra, además, que como puede actuar como única partícula introductora de discurso directo (29), aunque también se puede combinar, con menor frecuencia, con otros elementos, como los verbos decir, quedar, estar y sentir (30), la construcción y pronombre como (31), el adverbio ahi y la partícula onda (en su valor de operador de concreción).

29) H4A3: Entonces tú al final como que considerai cada cosa como una bolsa de palabras, como "ya, escribió tantas palabras y esto es".

30) H4A1: me dijo como "tengo hambre, que ganas de comprarme un completo"... 
31) M2A5: ... porque me decía, "no, ¿cómo no vamos a salir? Qué perna. ¿Cómo vai a estar leyendo el día domingo o el día sábado?", y yo como "pero es que me cuesta leer, me estoy acostumbrando de nuevo a la lectura, es diferente".

32) M7A4: Una vez del liceo nos mandaron a un jardín. En el jardín habían como treinta niñitos, igual era como-igual llegó un momento como que me desesperé, pero dije "ya, son niños" y me fui a sentar un rato, como que salí pa' afuera y entré y ahí como "ya".

33) M4A6: Claro y por ejemplo, a mí me dicen, onda como "y ahora qué, vai a estudiar algo, onda, si vai a estudiar informática, teníh que estudiar en la mejor universidad" así...

De esta manera, hemos podido realizar un primer acercamiento a la identificación de las combinatorias con las que como se presenta al introducir discurso. Con ello se abren nuevas posibilidades de estudio para el uso de esta partícula con esta función. En el apartado siguiente presentamos las principales conclusiones de este estudio y algunas proyecciones para este trabajo.

\section{CONCLUSIONES}

Con la realización de este estudio corroboramos el uso de como como partícula introductora de discurso directo en el español de Chile. Asimismo, hemos podido dar cuenta de que esta función conecta con y deriva del valor semántico aproximador de la partícula, dado que los datos demuestran que su uso contribuye a difuminar la literalidad del discurso introducido. Así, concluimos que como viene a marcar, a modo de pista, que el contenido que se reproduce no es exacto, sino aproximado.

En su calidad de partícula introductora de discurso directo, como permite introducir diferentes contenidos. En nuestro análisis hemos encontrado casos de habla directa propia y ajena, pensamientos de los propios hablantes, discurso estereotipado propio y ajeno, discurso hipotético propio y ajeno, interjecciones y sonidos. En este punto, creemos importante señalar que en otros trabajos se ha identificado además que como permite introducir gestos (Holmvik 2011); sin embargo, en nuestro corpus no hemos identificado este caso. Lo anterior creemos se debe a que hemos utilizado un corpus de trabajo en formato audio, por lo que, para estudiar la introducción de gestos mediante como se haría necesario contar con un corpus audiovisual. 
De los seis tipos de información introducidos en estilo directo por como que hemos identificado, consideramos importante destacar tanto el discurso estereotipado como el discurso hipotético, pues son contenidos que no habían sido reconocidos en estudios previos (Holmvik 2011; Jørgensen 2012; Panussis 2016; Mondaca 2017; Panussis y San Martín 2017; Mondaca 2019). Además, nuestro análisis ha dado cuenta de la relación que existe entre la reproducción de pensamientos propios e interjecciones y la expresión de actitudes, sentimientos y sensaciones de los hablantes. De esta forma, a través del análisis hemos podido observar que los hablantes plasman, en la reproducción de sus pensamientos y en la reproducción de interjecciones, cómo se sintieron/sienten o actuaron/actúan en determinadas situaciones.

En relación con aspectos sintácticos, nuestros resultados indican que como puede presentarse como único elemento introductor de discurso directo, aunque este no es el caso más frecuente. Así, cuando como opera con esta función, lo más habitual es que se posponga sintácticamente a otras formas, marcando la aproximación del discurso reproducido. En nuestro corpus, las combinatorias más frecuentes se dan con el adverbio así y con el verbo ser. Este primer acercamiento a la identificación de las combinatorias con las que como se presenta al introducir discurso directo abre algunas interrogantes, por ejemplo, ¿todas las combinatorias permiten introducir todos los tipos de información que hemos identificado? ¿Alguna de las combinatorias se vincula, preferentemente, con un tipo específico de información? Esbozamos, por tanto, como posibilidad de ampliación a este trabajo, un estudio en profundidad de las combinatorias de como como introductor de discurso directo, con el fin de responder a estas y otras interrogantes.

Finalmente, consideramos importante plantear la relevancia de un estudio que analice el uso de como con la función analizada en este trabajo y su relación con la atenuación pragmática. En nuestro corpus, hemos estudiado el valor aproximador de como y la introducción de discurso como función derivada; sin embargo, junto con ello, cabría estudiar si la introducción de estilo directo en como puede expresar en algunos contextos la intención de los hablantes de proteger su imagen ante posibles actos amenazadores. Asimismo, creemos interesante ampliar este análisis a la partícula como que, que, sobre la base de nuestros datos, también es utilizada como introductora de discurso directo y que, según se ha establecido en estudios anteriores, parece ser un indicador constante de atenuación (Panussis y San Martín 2017; Mondaca 2019). 


\section{REFERENCIAS BIBLIOGRÁFICAS}

ACAdemia Chilena de la lengua. 2010. Diccionario de uso del español de Chile. Santiago: MN Editorial.

ANDERSEN, GisLe. 2001. Pragmatic markers and sociolinguistic variation. A relevancetheoretic approach to the language of adolescents. Amsterdam/ Philadelphia: John Benjamins.

Borreguero, Margarita. 2017. Los relatos coloquiales: partículas discursivas y polifonía. Pragmalingüística 25: 62-88.

2020. Los marcadores de aproximación (en el lenguaje juvenil): esp. en plan vs. it. tipo. En M. A. Cuevas, F. Molina y P. Silvestri (coords.). España e Italia: un viaje de ida y vuelta. Studia in honorem Manuel Carrera Díaz, pp. 53-78. Sevilla: Editorial Universidad de Sevilla.

CAmargo, LaURa. 2007-2008. La cita como turno: el diálogo reconstruido en español oral desde la pragmática de corpus. Pragmalingüística 15-16: 49-70.

CAmEron, Richard. 1998. A Variable Syntax of Speech, Gesture, and Sound Effect: Direct Quotations in Spanish. Language Variation and Change 10 (1): 43-83.

De la Mora, Juliana. 2018. Las citas directas en el habla de la Ciudad de México. Anuario de Letras. Lingüística y Filología VI (2): 145-171.

GallucCI, María José. 2012a. Estilo directo e indirecto en interacciones orales: Estado de la cuestión en el ámbito hispánico. Boletín de Filología XLVII (2): 205-233.

2012b. Sintaxis de las citas en estilo directo e indirecto con verbo en el habla caraqueña. Lingüistica 28: 223-246.

2014. Entonces un muchacho llega y me dice: "Mira, regáleme un bolívar". Las citas en estilo directo e indirecto en el Corpus Diacrónico del Habla de Caracas 1987/2013. Boletin de Lingüistica XXVI (41-42): 45-74.

2016. El discurso referido en los manuales sobre análisis del discurso y pragmática lingüística. Lengua y Habla 20: 200-224.

Grutschus, ANKe. En prensa. Y yo en plan: “QQué es esto?” Los marcadores de cita en el español coloquial. Spanish in Context.

Haspelmath, Martin y Oda BuchHolz. 1998. Equative and Similative Constructions in languages of Europe". En Johan Van der Auwera (ed.). Adverbial Constructions in the Languages of Europe, pp. 277-334. Berlín/Nueva York: Mouton de Gruyter.

Herrero Cecilia, Juan. 2006. Teorías de pragmática, de lingüistica textual y de análisis del discurso. Cuenca: Ediciones de la Universidad de Castilla-La Mancha.

Holmvik, Lise. 2011. Como usado como marcador del discurso en el lenguaje juvenil de Madrid. Tesis para optar al grado de Máster en el Departamento de Lenguas Extranjeras. Universidad de Bergen.

Jørgensen, Annette Myre. 2009. En plan used as a hedge in Spanish teenage language. En Anna-Brita Stenström y Annette Myre Jørgensen, Youngspeak in a Multilingual Perspective. Pragmatics \& Beyond New Series 184, pp. 95-115. Amsterdam/Philadelphia: John Benjamins Publishing Company.

Y ANNA-BRITA STENSTRÖM. 2009. Dos marcadores pragmáticos contrastados en el lenguaje juvenil: el inglés like y el español como. Español Actual 92: 103-120.

2012. Funciones del marcador pragmático como en el lenguaje juvenil español y chileno. En María Elena Placencia y Carmen García (eds.). Pragmática y comunicación intercultural en el mundo hispanohablante, pp. 209-230. Londres: Rodopi.

Levey, STEPHEN. 2003. He's like 'Do it now!' and I'm like 'No!'. Some innovative quotative usage among young people in London. English Today 73, 19 (1): 24-32. 
López Alonso, Covadonga. 2014. Análisis del Discurso. Madrid: Síntesis.

MÉNDEZ, MARÍA. 2016. Valores pragmático-discursivos de la construcción lingüística en plan. ¿Formación de un nuevo marcador? Philologia Hispalensis 30 (1): 123-144.

Minatsch, Wiltrud. 2009. The Approximators French Comme, Italian Come, Portuguese Como and Spanish Como from a Grammaticalization Perspective. En Corinne Rossari, Claudia Ricci y Adriana Spiridon (eds.). Grammaticalization and Pragmatics: Facts, Approaches, Theoretical Issues, pp. 65-91. Bingley: Emerald Group Publishing Limited. 2010. Sincronía y diacronía del aproximador "como". Revista internacional de lingüística iberoamericana 16: 175-201.

2018. De la escritura científica a la conversación coloquial adolescente. El caso de tipo. Spanish in Context 15 (2): 281-304.

2020. Los orígenes discursivos de los atenuadores procedimentalizados 'tipo', 'onda', 'corte' y 'rollo': Una exploración microdiacrónica. Revista Signos. Estudios de Lingüística 53 (104): 686-717.

MonDACA, LisSETTE. 2017. La atenuación por medio del marcador discursivo conversacional como en el español de Chile. Tesis para optar al grado de Máster en Estudios del Discurso: Comunicación, Sociedad y Aprendizaje. Universitat Pompeu Fabra.

2019. Approximators and mitigators in Chilean Spanish: the case of 'como' and 'como que'. Texts in Process 5 (1): 29-52.

2020. Aproximación al estudio de las funciones pragmáticas y a la atenuación en la partícula 'onda' en el español de Chile. Revista Signos. Estudios de Lingüística 53 (104): 718-743.

Panussis, Constanza. 2016. Análisis pragmático y sociolingüístico de las funciones del marcador como (que) en el habla de Santiago de Chile. Informe final de Seminario para optar al grado de Licenciada en Lengua y Literatura Hispánica con Mención en Lingüística, Universidad de Chile.

y Abelardo San Martín. 2017. Como (que) y sus funciones discursivas en el habla santiaguina: análisis pragmático y sociolingüístico. RLA, Revista de Lingüística Teórica y Aplicada 55 (2): 39-61.

PortolÉs, José. 2004. Pragmática para hispanistas. Madrid: Síntesis.

Real Academia Española. 2010. Nueva Gramática de la Lengua Española: Manual. Madrid: S.L.U. Espasa Libros.

2021. Diccionario de la lengua española. [en línea].

Rodríguez, LAURA. 2015. Marcador conversacional en plan en el habla actual de Galicia. Trabajo para optar al Grado en Lengua y Literatura Españolas. Universidad de Santiago de Compostela.

ROJAS, DARÍo. 2008. Funciones actuales y evolución semántica de la locución de repente en el español de Chile. Boletín de Filología XLIII: 207-237.

San Martín, Abelardo y Silvana Guerrero. 2013. Una aproximación sociolingüística al empleo del discurso referido en el corpus PRESEEA de Santiago de Chile. Signos, 46 (82): 258-282.

, Cristian Rojas y Silvana Guerrero. 2016. La función discursiva y la distribución social de los marcadores por ser y onda en el corpus del PRESEEA de Santiago de Chile. Boletín de Filología LI (2): 235-254.

Tagliamonte, Sali. 2009. Be like: The New Quotative in English. En N. Coupland y A. Jaworski (eds.). The New Sociolinguistics Reader, pp. 75-91. New York: Palgrave Macmillan. Press.

2016. Teen Talk. The Language of Adolescents. Cambridge: Cambridge University 
Voghera, Miriam. 2013. Da nome tassonomico a segnale discorsivo: una mappa delle costruzioni Di tipo in italiano contemporaneo. Studi di grammatica italiana 33: 197-221.

Wagner, Claudio. 1998. El atlas lingüístico y etnográfico de Chile por regiones (ALECh). Estudios Filológicos 33: 119-129.

2004. El atlas lingüístico y etnográfico de Chile. Localidades y cuestionario. Estudios Filológicos 39: 83-120. 